\title{
On the universality of inertial energy in the log layer of turbulent boundary layer and pipe flows
}

\author{
D. Chung · I. Marusic · J. P. Monty · M. Vallikivi • A. J. Smits
}

Received: date / Accepted: date

\begin{abstract}
Recent experiments in high Reynolds number pipe flow have shown the apparent obfuscation of the $k_{x}^{-1}$ behaviour in spectra of streamwise velocity fluctuations (Rosenberg et al, 2013). These data are further analysed here from the perspective of the $\log r$ behaviour in second-order structure functions, which have been suggested as a more robust diagnostic to assess scaling behaviour. A detailed comparison between pipe flows and boundary layers at friction Reynolds numbers of $R e_{\tau} \approx 5000$ to 20000 reveals subtle differences. In particular, the $\log r$ slope of the pipe-flow structure function decreases with increasing wall distance, departing from the expected $2 A_{1}$ slope in a manner that is different to boundary layers. Here, $A_{1} \approx 1.25$, the slope of the log law in the streamwise turbulence intensity profile at high Reynolds numbers. Nevertheless, the structure functions for both flows recover the $2 A_{1}$ slope in the log layer sufficiently close to the wall, provided the Reynolds number is also high enough to remain in the log layer. This universality is further confirmed in very high Reynolds number data from measurements in the neutrally stratified atmospheric surface layer. A simple model that accounts for the 'crowding' effect
\end{abstract}

D. Chung, I. Marusic, J. P. Monty

Department of Mechanical Engineering,

University of Melbourne,

Victoria 3010, Australia

E-mail: daniel.chung@unimelb.edu.au

M. Vallikivi, A. J. Smits

Department of Mechanical and Aerospace Engineering,

Princeton University,

Princeton, NJ 08544, USA

A. J. Smits

Department of Mechanical and Aerospace Engineering,

Monash University,

Victoria 3800, Australia near the pipe axis is proposed in order to interpret the aforementioned differences.

Keywords turbulent pipe flow · turbulent boundary layers · boundary layer structure

\section{Introduction}

Scaling of the spectrum of velocity fluctuations in wall turbulence is of great interest since this characterises the behaviour of individual scales and, therefore, directly informs us about the physical structure of the turbulence. Theoretical bases for spectral scalings are few. However, in the inertial range where direct viscous effects are unimportant, application of dimensional analysis and the notion of a spectral overlap leads to the prediction that spectra follow a $k_{x}^{-1}$ dependence in this range (Perry and Abell, 1975; Perry et al, 1986), written as

$\frac{E_{u u}\left(k_{x}\right)}{U_{\tau}^{2}}=\frac{A_{1}}{k_{x}}$.

Here, $E_{u u}\left(k_{x}\right)$ is the one-dimensional (1D) power spectral density of the streamwise velocity fluctuation, normalised such that $\overline{u^{2}}=\int_{0}^{\infty} E_{u u}\left(k_{x}\right) \mathrm{d} k_{x}, k_{x}$ is the streamwise wavenumber and $A_{1}$ is a universal constant named after Townsend and Perry (Townsend, 1976; Perry et al, 1986; Marusic et al, 2013). The scaling (1) is for the large anisotropic inertial scales, not to be confused with the $k_{x}^{-5 / 3}$ scaling for the small isotropic inertial scales. In this paper $x, y$ and $z$ are the streamwise, spanwise and wall-normal coordinates, respectively, and $U_{\tau}$ is the friction velocity. This $k_{x}^{-1}$ formulation is consistent with the attached-eddy hypothesis (Townsend, 1976; Perry and Chong, 1982), although it is noted that other theories arrive at the same result (e.g. Högström et al, 

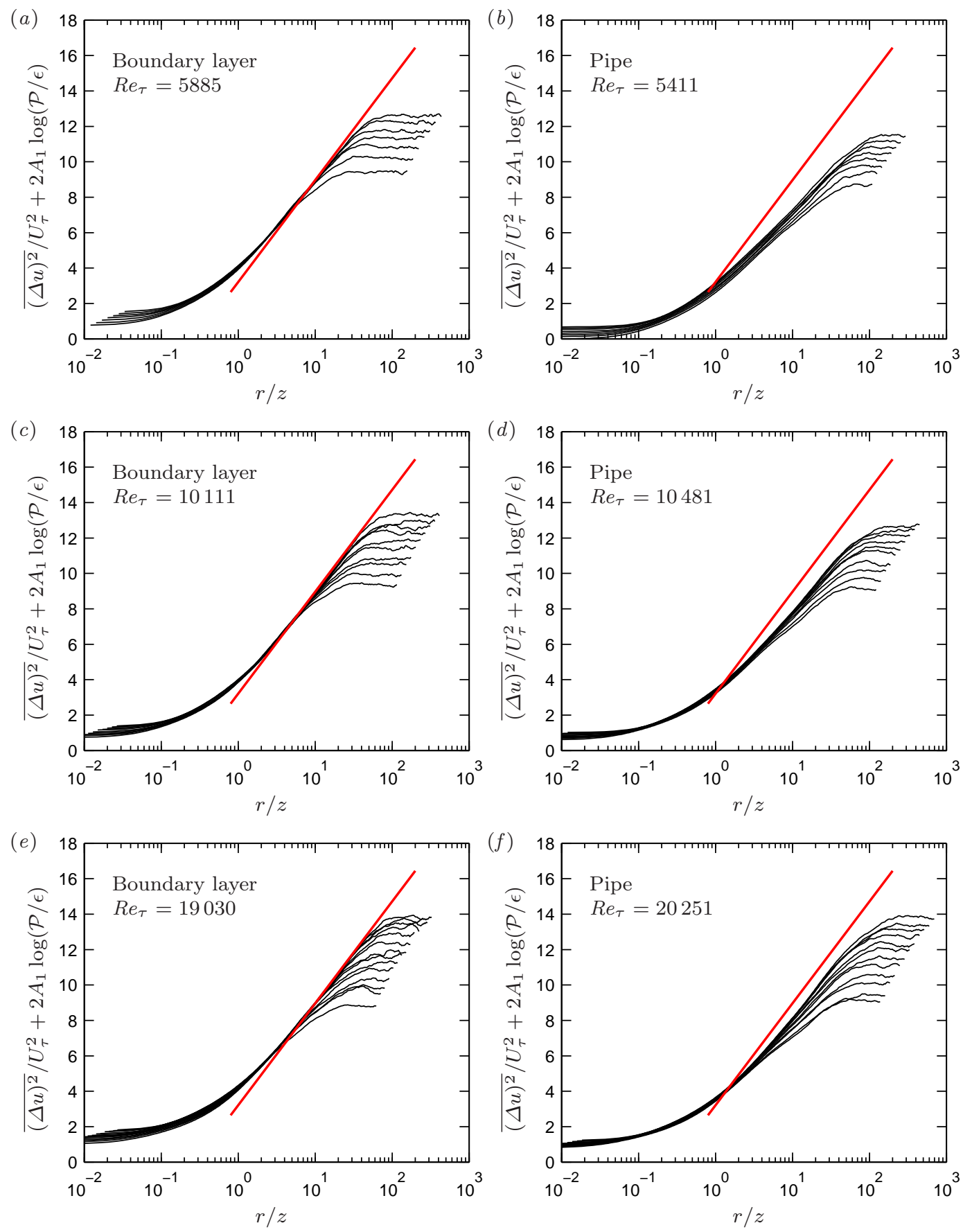

Fig. 1 Second-order structure function plotted against longitudinal separation, $r$, scaled with wall-distance, $z$, in the log region of: turbulent boundary layer at $(a) R e_{\tau}=5890,(c) R e_{\tau}=10110$ and (e) $R e_{\tau}=19030$ (Hutchins et al, 2009; Kulandaivelu, 2012 ) and turbulent pipe flow at $(b) R e_{\tau}=5410,(d) R e_{\tau}=10480$ and $(f) R e_{\tau}=20250$ (Rosenberg et al, 2013). The structure function is corrected for the imbalance between production and dissipation (Davidson and Krogstad, 2009). The value of the structure function as $r / z \rightarrow \infty$ decreases with increasing wall-distance, $z$. In all figures the solid red line represents a $\log$ law with slope $2 A_{1}=2.5$. 

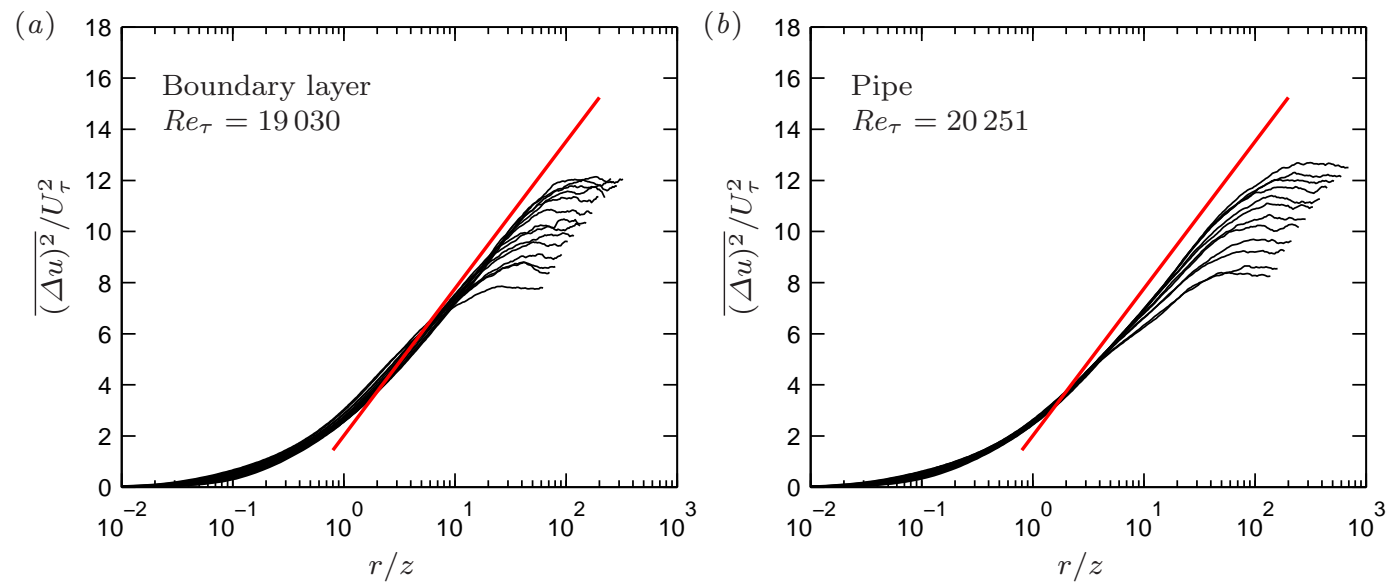

Fig. 2 Second-order structure function, without the production-to-dissipation correction, plotted against longitudinal separation, $r$, scaled with wall-distance, $z$, in the log region of: turbulent boundary layer at $(a) R e_{\tau}=19030$ (Hutchins et al, 2009 ) and turbulent pipe flow at $(b) R e_{\tau}=20251$ (Rosenberg et al, 2013). The value of the structure function as $r / z \rightarrow \infty$ decreases with increasing wall-distance, $z$. In all figures the solid red line represents a log law with slope $2 A_{1}=2.5$.

2002; Davidson and Krogstad, 2009). In general, this scaling is independent of the spectral-overlap argument and expected if, at a given wall-distance $z$, there is similarity of the turbulent flow between the large $\left(\Delta_{E}\right)$ eddies down to the locally scaled $(z)$ eddies. Indeed Vallikivi et al $(2015 \mathrm{a}, \mathrm{b})$ have shown no evidence of spectral overlap in high Reynolds number boundary layers or pipe flows. Here, $\Delta_{E}$ denotes either the pipe radius, $R$, or the boundary-layer thickness, $\delta$. Simply put, $A_{1}=k_{x} E_{u u}\left(k_{x}\right) / U_{\tau}^{2}$ is the only dimensionless group once $z$ and $\Delta_{E}$ are discounted.

The highest Reynolds number wall-turbulence measurements in a laboratory are those acquired in the Princeton Superpipe (Hultmark et al, 2012). From an analysis of these data, Rosenberg et al (2013) presented premultiplied 1D spectra of streamwise velocity fluctuations at $R e_{\tau}=R^{+} \approx 2000-100000$, where ' + ' denotes viscous scaling (the viscous length and velocity scales are $\nu / U_{\tau}$ and $U_{\tau}$, respectively, where $\nu$ is the kinematic viscosity). The spectra do not exhibit the $k_{x}^{-1}$ region expected at such high Reynolds numbers where sufficient scale separation should exist. In fact, the energy associated with the $k_{x}^{-1}$ range of scales appears to decrease with increasing wall-distance in pipe flow, an effect Rosenberg et al (2013) attributed to the increasing influence of large scales. This is in contrast to the observations by Nickels et al (2005) in a boundary layer at $R e_{\tau}=\delta^{+} \approx 14380$ where $k_{x}^{-1}$ scaling was reported for about a third of a decade at $z^{+} \approx 100$. Despite these apparent differences in spectral scaling, the integral of the spectrum, which is the turbulence intensity, appears to follow the same-sloped log law in the log region of both flows where $A_{1} \approx 1.25$ (Hultmark et al, 2012; Marusic et al, 2013).
Davidson et al (2006b) argue that the 1D spectrum of streamwise velocity is not the ideal diagnostic tool to investigate the behaviour of, or indeed, find, the $k_{x}^{-1}$ range due to aliasing. The aliasing comes from the fact that the 1D spectrum is the integral of the 2D spectrum, such that energy from a large range of spanwise length scales contributes to the energy of a single streamwise length scale. Davidson et al (2006b) suggest that the second-order structure function is a better diagnostic. These authors show that the $k_{x}^{-1}$ behaviour of the 1D spectrum corresponds to $\log r$ behaviour of the structure function,

$$
\frac{\overline{(\Delta u)^{2}}(r)}{U_{\tau}^{2}}=2 B_{1}+2 A_{1} \log \left(\frac{r}{z}\right)
$$

where $r$ is the spatial separation in the streamwise direction and $B_{1}$ is a constant that depends on flow geometry. Davidson et al (2006a) extend this work to include a term for the imbalance between turbulent energy production $\mathcal{P}$ and dissipation $\epsilon$,

$$
\frac{\overline{(\Delta u)^{2}}(r)}{U_{\tau}^{2}}=2 B_{1}+2 A_{1} \log \left(\frac{r}{z}\right)-2 A_{1} \log \left(\frac{\mathcal{P}}{\epsilon}\right),
$$

and show excellent agreement with high Reynolds number turbulent boundary layer data. In the following, we will examine the structure function for turbulent pipe and boundary-layer flows, and show that the aforementioned differences between these flows can be reconciled, provided $z$ is chosen to be sufficiently close to the wall, while at the same time maintaining a sufficiently high Reynolds number. When both these conditions are met, the $\log r$ region exhibits a slope corresponding to $A_{1} \approx 1.25$ independent of the flow geometry. 
$(a)$

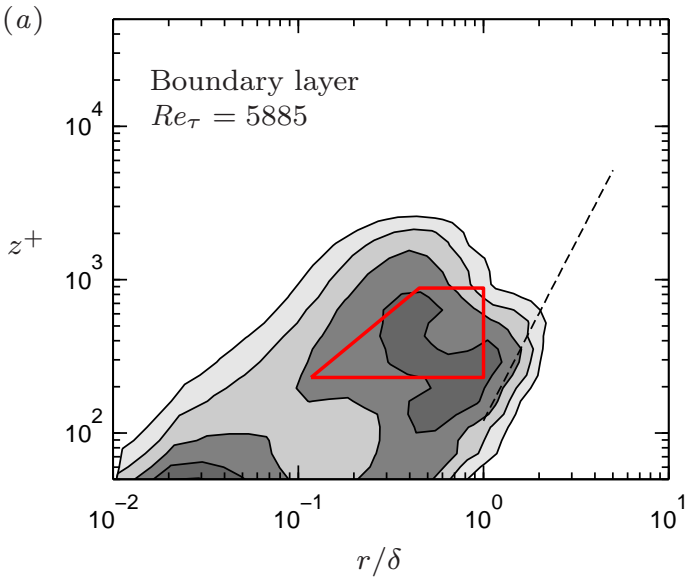

(c)

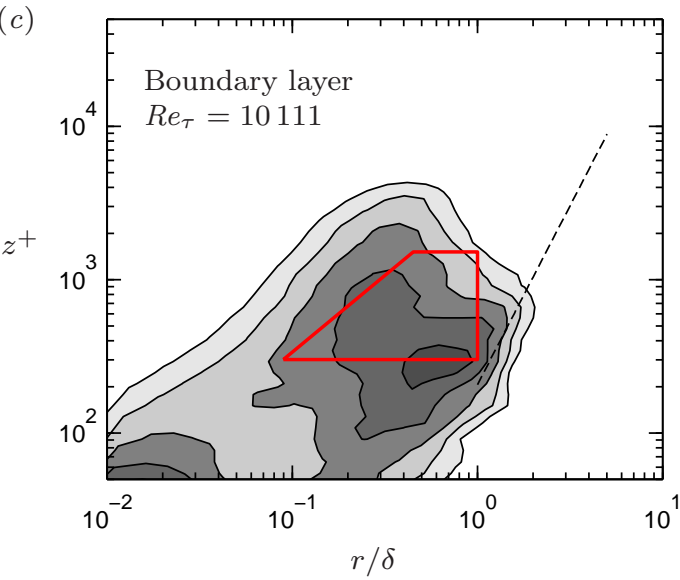

$(e)$

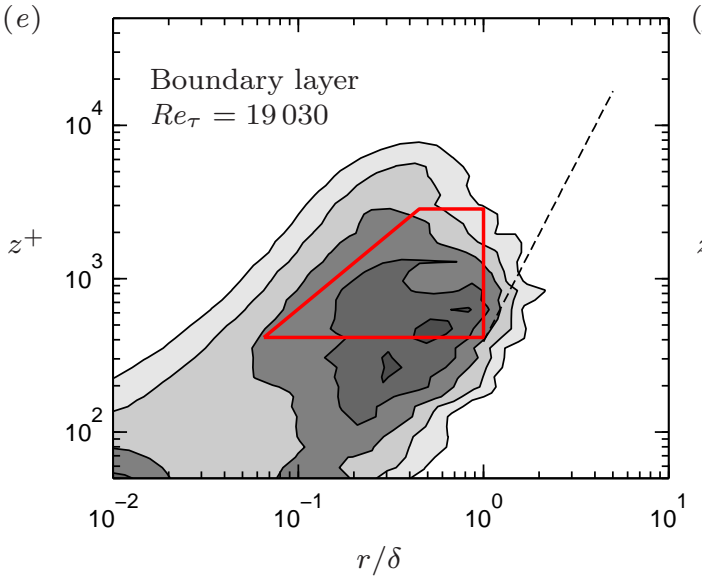

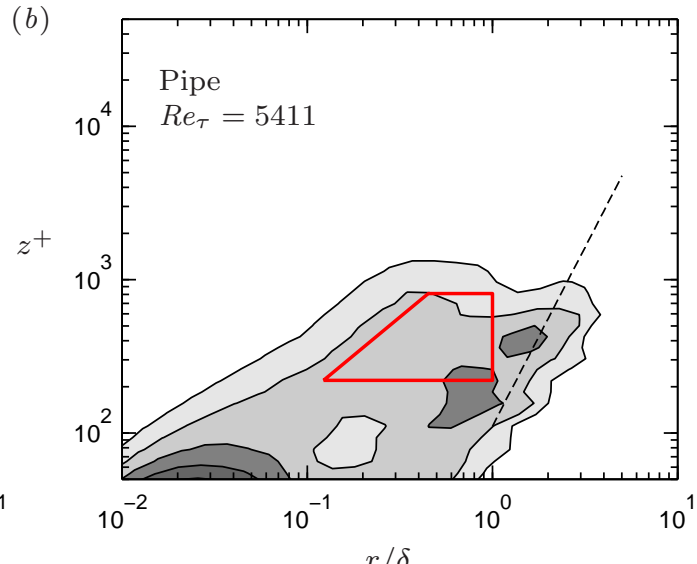

$(d)$
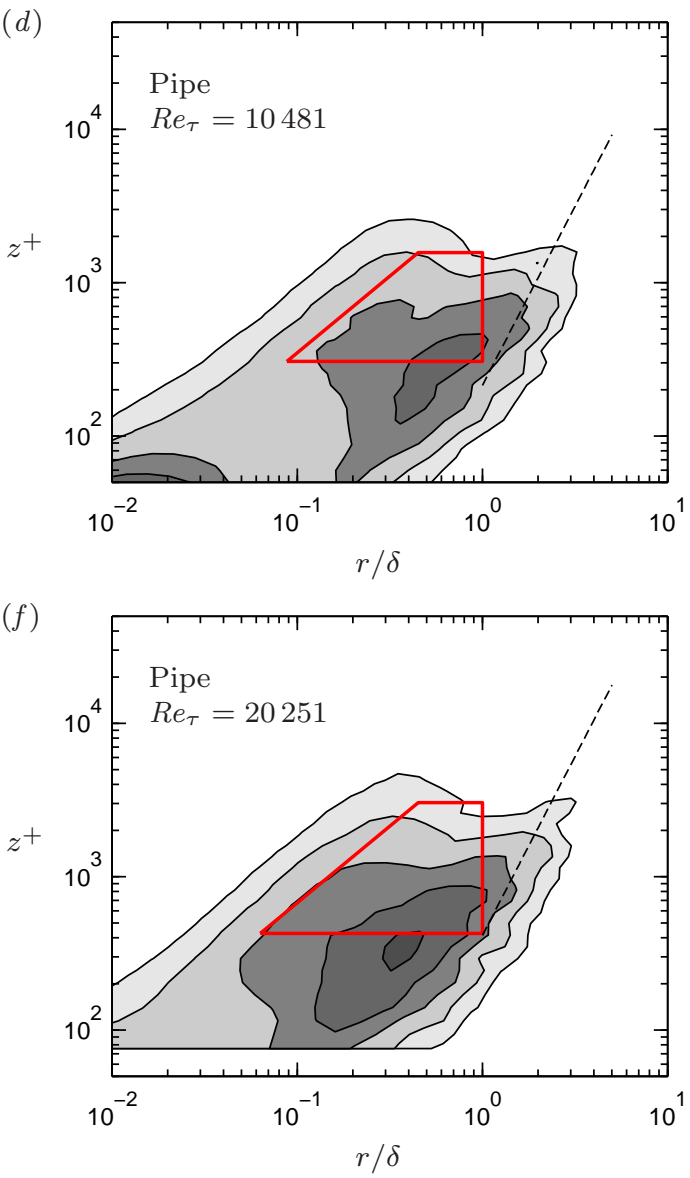

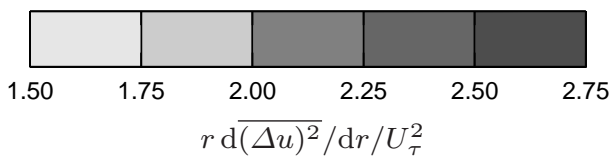

Fig. 3 Premultiplied second-order structure-function maps for both boundary layers and pipes at various Reynolds numbers. Data are as described in figure 1. The thick solid red line demarcates the region, $3 z<r<\Delta_{E}, 3 \operatorname{Re}_{\tau}^{1 / 2}<z^{+}<0.15 R e_{\tau}$, where $r \mathrm{~d} \overline{(\Delta u)^{2}} / \mathrm{d} r / U_{\tau}^{2} \rightarrow 2 A_{1} \approx 2.5$ is expected. The three highest (darkest) colours correspond to $2.5 \pm 20 \%$. The thin dashed black line traces the very-large-scale motions (VLSMs), given by $r / \delta \propto(z / \delta)^{3 / 7}$ (Monty et al, 2009), here $r / \delta=5.3(z / \delta)^{3 / 7}$. 


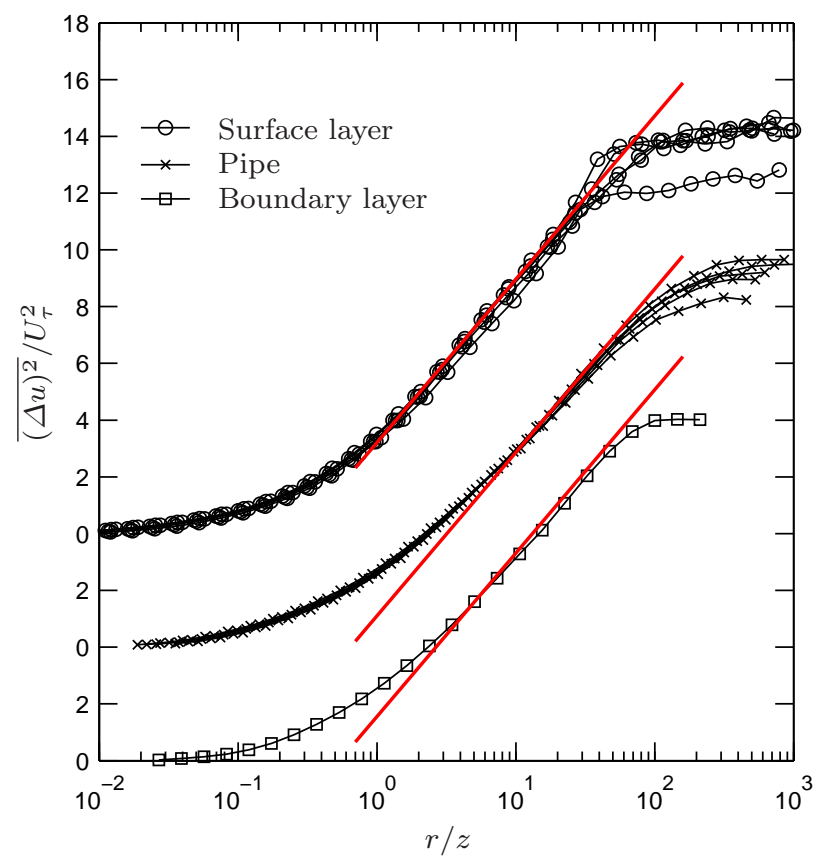

Fig. 4 High Reynolds number second-order structure functions in the log region for $z / \Delta_{E}<0.025$ from the Melbourne wind tunnel (Hutchins et al, 2009) at $R e_{\tau}=19030$; the Princeton Superpipe (Rosenberg et al, 2013) at $R e_{\tau}=98190$; and the SLTEST atmospheric surface layer (Kunkel and Marusic, 2006) at $R e_{\tau} \approx 10^{6}$ all show at least a decade of logarithmic region approaching the same slope, $2 A_{1}=2.5$ (thick solid red line). Each set of curves is shifted upwards by four units for legibility.

\section{Structure functions of boundary layer and pipe flow}

Figure 1 displays the second-order structure function for turbulent boundary layers and pipe flows at nominally matched Reynolds numbers. The turbulent boundary layer data are from the University of Melbourne High Reynolds Number Boundary Layer Wind Tunnel (Hutchins et al, 2009; Kulandaivelu, 2012) and the turbulent pipe flow data are from the Princeton $\mathrm{Su}-$ perpipe (Hultmark et al, 2012). The precise bounds of the overlap or classical logarithmic region in physical space remains an open topic of debate. For example, classically the start of the log region is taken to be at a fixed value of $z^{+}$, while others (e.g. Klewicki et al, 2009) have argued that it scales with $R e_{\tau}^{1 / 2}$ in inner variables. Throughout this paper we will follow Marusic et al (2013) and refer to the 'log region' as the range of walldistances where the mean and variance of the streamwise velocity follow logarithmic laws, and use the tentative and conservative limits, $3 R e_{\tau}^{1 / 2}<z^{+}<0.15 R e_{\tau}$. This choice of limits does not affect the general conclusions drawn in this paper.

Note that in figure 1 the correction for the productiondissipation imbalance has been added to the structure function in the figure (Davidson et al, 2006a). The turbulent boundary layer data display excellent collapse onto the expected log law and the data systematically peel off from this log law to a constant level at an $r / z$ value that reduces with increasing wall-distance. However, the pipe flow data display a subtle but noticeably different behaviour. The data in figure $1(b, d, f)$ could be interpreted as either peeling off from a log law at a lower $r / z$ (for a given wall-distance) or the slope of the structure function varies with wall-distance. At the highest Reynolds number, the latter interpretation seems more accurate. Interestingly, as the wall is approached, the data fall back on to the same log law that describes the turbulent boundary layer data at the highest Reynolds number (figure $1 e, f$ ). Further, the steady march of the pipe structure functions towards the $2 A_{1}$ slope (red line) for the two lower Reynolds numbers (figure $1 b, d$ ) appears to be halted due to the shorter extent of the log region.

In figure 2, we show the same data for the highest Reynolds numbers without the production-to-dissipation correction to demonstrate the effect of the correction. Some differences can be seen in the $z$-dependent shifts relative to figure 1 , but the systematic departure from the $2 A_{1}$ slope at large wall distances (the lower curves) of pipe flow relative to the boundary layer remains clear. Perhaps a more searching test is in the premultiplied second-order structure function, that is, $r \mathrm{~d} \overline{(\Delta u)^{2}} / \mathrm{d} r / U_{\tau}^{2}$, in which a flat region with level $2 A_{1}$ is expected in the $\log$ region between $z \ll r \ll \Delta_{E}$. The production-todissipation shift does not influence this diagnostic. In 
(a)

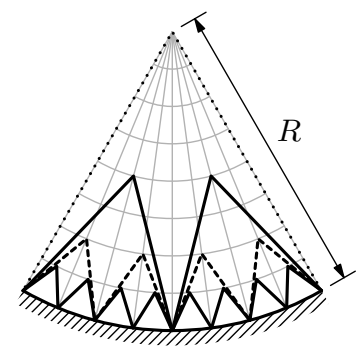

(b)

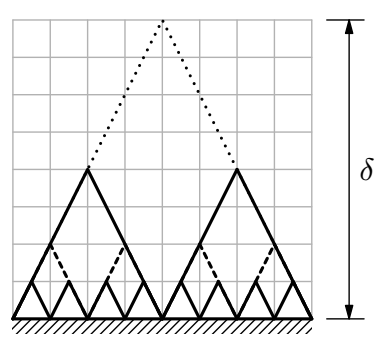

Fig. 5 Hierarchy of eddies in the cross plane highlighting geometrical differences between $(a)$ a pipe and $(b)$ a boundary layer, adapted from Monty (2005).

figure 3, contours of this diagnostic are shown, with the wall-distance $z$ mapped onto the ordinate. Here, we demarcate the region bounded by $3 z<r<\Delta_{E}$. The three highest (darkest) contour levels denote $2.5 \pm 20 \%$. A relatively inclusive error bar is used here because of difficulties with differentiating experimental data. At the lowest Reynolds number, $R e_{\tau} \approx 5000$, the pipe-flow data show only a small region close to the wall in the log layer that reaches the $2.5 \pm 20 \%$ level in contrast to the boundary-layer data which shows a substantial region that reaches the same level. At the highest Reynolds number, $R e_{\tau} \approx 20000$, the region close to the wall in pipe flow in the log layer becomes stronger but a systematic reduction in $r \mathrm{~d} \overline{(\Delta u)^{2}} / \mathrm{d} r / U_{\tau}^{2}$ remains with increasing wall distance. For reference, we also show in figure 3 the line corresponding to $r / \delta \propto(z / \delta)^{3 / 7}$, which has previously been documented as a signature of the very-large-scale motions (VLSMs) (Monty et al, 2009). Consistent with Monty et al (2009), we observe that the VLSMs are more energetic in pipe flows (whose premultiplied structure functions trace extended ridges along this line to lengths in excess of $20 R$ ) than in boundary layers.

We provide further evidence of the universality of the $2 A_{1}$ constant of the $\log r$ region by compiling very high Reynolds number data from the Melbourne wind tunnel, the SLTEST atmospheric surface layer and the Princeton Superpipe at available log-layer locations closest to the wall (figure 4). These data, all of which satisfy $z / \Delta_{E}<0.025$ show at least a decade of $2 A_{1}$ slope in the structure function. Noticeable deviations from this $\log$ behaviour for $\overline{(\Delta u)^{2}}$ appear for the pipe results for higher $z / \Delta_{E}$ values, even though the mean flow and $\overline{u^{2}}$ results follow log behaviour up until $z / \Delta_{E} \approx 0.15$.

\section{Discussion}

The similarity between the boundary layer and pipe flow at distances sufficiently close to the wall, where geometrical differences are negligible, suggests a geo-

metrical explanation. The variations of the $\log r$ slope with wall-distance could be attributed to the restricted size of eddies due to the confinement of the pipe geometry, a restriction that is absent in the boundary layer. We consider this interpretation in the discussion that follows. In boundary layers, a number of different characteristic shapes have been used in attached-eddy modelling in the literature (Perry et al, 1986). For illustrative purposes, consider the simplest type of eddy with a $\Lambda$ shape in figure $5(a)$, as used by Monty (2005). A basic tenet of the attached-eddy model is that there exist hierarchies of geometrically similar representative eddies. However, the geometry of the pipe does not strictly permit such hierarchies when the eddy size becomes a significant fraction of the pipe radius, $R$, as can be seen in the comparison of figures $5(a, b)$. Further, it is known from two-dimensional potential flow that a point vortex at $z$ in a circle has its inverse (image) point at $-z /(1-z / R)=-z+O(z / R)$; while for a flat plate, the image is simply located at $-z$. A comparison of these two image-vortex systems suggest a geometrical correction that depends on $z / R$ and that the correction is negligible for vanishing $z / R$, i.e. at distances sufficiently close to the wall. It can also be observed that, assuming the same eddy construction, the available space per wall-parallel area in pipe flow is less than that of a boundary layer, and this 'crowding' effect becomes more pronounced with increasing wall distance. Unlike the boundary layer in which the width is unbounded, the geometry of the pipe imposes a maximum width, $\left(\lambda_{y}\right)_{\max }=2 \pi(R-z)$. These considerations suggest that the inertial scales in pipe flow cannot be expected to be entirely similar to its boundary-layer counterpart if $z$ is not sufficiently small relative to $R$, as observed in the structure functions presented in $\S 2$.

In $\S 3.2$, we propose to model this 'crowding' effect by simply reducing the upper limit of the widest eddies in the two-dimensional spectrum. First, we lay the groundwork in $\S 3.1$ for this interpretation by revisiting the arguments leading to the $k_{x}^{-1}$ range in the context of one- and two-dimensional spectra of turbulent boundary layers. We then show in $\S 3.3$ that the postulated pipe geometrical effects show behaviours that are consistent with the data presented in $\S 2$.

\subsection{One- and two-dimensional spectra}

Following del Álamo et al (2004), let us consider the two-dimensional (2D) spectrum, $E_{u u}^{2 D}\left(k_{x}, k_{y}\right)$, in the inertial region. Dimensional considerations suggest that there is a range of scales over which $k_{x} k_{y} E_{u u}^{2 D}\left(k_{x}, k_{y}\right) / U_{\tau}^{2}$ is approximately constant $(\approx 1$ according to del Álamo et al, 2004). Let this region of constancy be bounded 
by the limits $\lambda_{y} / z=\lambda_{L} / z=C_{1} f_{1}\left(\lambda_{x} / z\right)$ and $\lambda_{y} / z=$ $\lambda_{U} / z=C_{2} f_{2}\left(\lambda_{x} / z\right)$, where $f_{1}(1)=f_{2}(1)=1$, as illustrated in figure 6 . The wavelength, $\lambda$, is inversely proportional to the wavenumber, $k$ (e.g. $\lambda_{y}=2 \pi / k_{y}$ ). Without detailed knowledge of the spectrum at high Reynolds numbers, the precise forms of $\lambda_{L}$ and $\lambda_{U}$ are unknown but we expect that $C_{1}<1$ and $C_{2}>1$. Given an eddy of streamwise length $\lambda_{x},\left(C_{1}, C_{2}\right)$ is the range of eddy widths that are self similar. We allow the eddy to have widths ranging from less than its length $\left(C_{1}<1\right)$ to greater than its length $\left(C_{2}>1\right)$. These expectations are only stated to give some physical insight; they are not necessary for the following analysis. Physically, these conditions mean that eddies of scale $z$ and aspect ratio $\lambda_{x} / \lambda_{y}=1$ are included in the region where $k_{x} k_{y} E_{u u}^{2 D}\left(k_{x}, k_{y}\right) / U_{\tau}^{2}$ is approximately constant. In figure 6 , the upper and lower bounds are straight lines on a $\log -\log$ plot, such that $f_{1}$ and $f_{2}$ are power laws; in the figure, $f_{1}\left(\lambda_{x} / z\right)=f_{2}\left(\lambda_{x} / z\right)=\left(\lambda_{x} / z\right)^{1}$. Note that figure 6 is for illustrative purposes; $f_{1}$ and $f_{2}$ can be general functions in the following analysis, but are most likely to be power laws (del Álamo et al, 2004).

The $1 \mathrm{D}$ spectrum for this range of scales is calculated by integration of the $2 \mathrm{D}$ spectrum:

$$
\frac{k_{x} E_{u u}\left(k_{x}\right)}{U_{\tau}^{2}}=\int_{\lambda_{L}}^{\lambda_{U}} \frac{k_{x} k_{y} E_{u u}^{2 D}\left(k_{x}, k_{y}\right)}{U_{\tau}^{2}} \frac{d \lambda_{y}}{\lambda_{y}}
$$

Technically, integration over the negative wavelengths should also be included, but is unnecessary for the argument at hand. For simplicity, we will assume that $k_{x} k_{y} E_{u u}^{2 D}\left(k_{x}, k_{y}\right) / U_{\tau}^{2} \approx 1$ between these limits (del Álamo et al, 2004) so that the integral is easily evaluated as

$$
\frac{k_{x} E_{u u}\left(k_{x}\right)}{U_{\tau}^{2}}=\log \left(\frac{\lambda_{U}}{\lambda_{L}}\right)=\log \left(\frac{C_{2} f_{2}\left(\lambda_{x} / z\right)}{C_{1} f_{1}\left(\lambda_{x} / z\right)}\right) .
$$

Now, to recover the traditional $k_{x}^{-1}$ scaling of the $1 \mathrm{D}$ energy spectrum, the functions $f_{1}$ and $f_{2}$ may remain arbitrary, but must be identical such that the righthand side of (5) is a constant. If $f_{1}\left(\lambda_{x} / z\right)=f_{2}\left(\lambda_{x} / z\right)$ then

$$
\frac{k_{x} E_{u u}\left(k_{x}\right)}{U_{\tau}^{2}}=\log \left(\frac{C_{2}}{C_{1}}\right)=A_{1},
$$

which is the $k_{x}^{-1}$ scaling with the Townsend-Perry constant $A_{1} \approx 1.25$. Note that differences in the functional forms between $f_{1}$ and $f_{2}$ will result in variations from the $k_{x}^{-1}$ dependence (see del Álamo et al, 2004). Such variations are beyond the scope of this paper. It should be noted that the conclusions drawn are not dependent on $f_{1}$ and $f_{2}$ being equivalent (i.e. on the existence of $k_{x}^{-1}$ ) as discussed later.

The turbulence intensity is simply the integral of the streamwise 1D energy spectrum. Including contributions to the spectrum from large wavelengths that

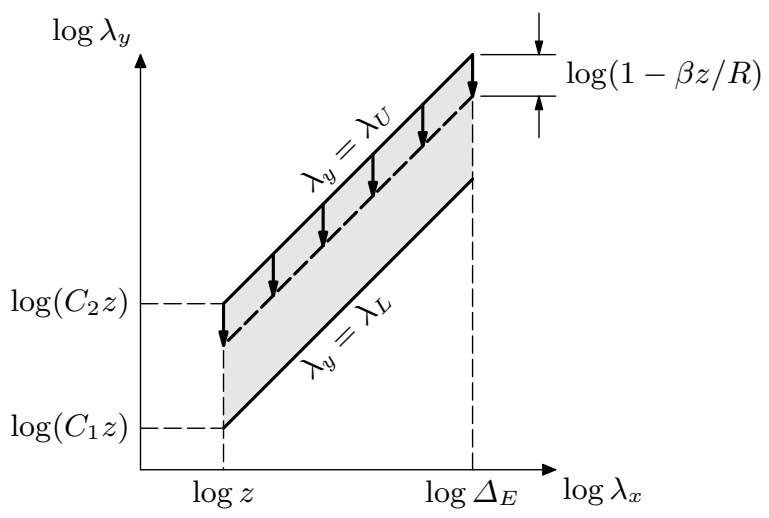

Fig. 6 Illustration of the $2 \mathrm{D}$ spectrum, where the grey area bounded by the lines, $\lambda_{L} / z=C_{1} f_{1}\left(\lambda_{x} / z\right)$ and $\lambda_{U} / z=$ $C_{2} f_{2}\left(\lambda_{x} / z\right)$, is the region where $k_{x} k_{y} E_{u u}^{2 D}\left(k_{x}, k_{y}\right) / U_{\tau}^{2}$ is approximately constant. The constraining effect of the pipe geometry is modelled as a blockage that reduces the width of eddies, $\lambda_{U}$, by a factor $(1-\beta z / R)$, where $\beta>0$ is a model constant, i.e. the crowding effect more strongly felt near the pipe axis, where $z / R$ is larger.

scale with $\Delta_{E}$, and also small but inertial wavelengths that scale with $z$, the turbulence intensity in the $\log$ region is given by

$$
\begin{aligned}
\frac{\overline{u^{2}}}{U_{\tau}^{2}}= & \int_{0}^{\infty} \frac{E_{u u}\left(k_{x}\right) d k_{x}}{U_{\tau}^{2}}=\int_{0}^{1 / \Delta_{E}} \frac{E_{u u}\left(k_{x}\right)}{U_{\tau}^{2}} \mathrm{~d} k_{x} \\
& +\int_{1 / \Delta_{E}}^{1 / z} \frac{E_{u u}\left(k_{x}\right)}{U_{\tau}^{2}} \mathrm{~d} k_{x}+\int_{1 / z}^{\infty} \frac{E_{u u}\left(k_{x}\right)}{U_{\tau}^{2}} \mathrm{~d} k_{x} \\
\sim & \int_{0}^{1} g_{1}\left(k_{x} \Delta_{E}\right) \mathrm{d}\left(k_{x} \Delta_{E}\right)+\int_{1 / \Delta_{E}}^{1 / z} \frac{A_{1}}{k_{x}} \mathrm{~d} k_{x} \\
& +\int_{1}^{\infty} g_{2}\left(k_{x} z\right) \mathrm{d}\left(k_{x} z\right) \sim B_{1}-A_{1} \log \left(\frac{z}{\Delta_{E}}\right),
\end{aligned}
$$

where $g_{1}\left(k_{x} \Delta_{E}\right)$ represents the normalised spectrum of the large wavelengths, $g_{2}\left(k_{x} z\right)$ represents the normalised spectrum of the small but inertial wavelengths and $B_{1}$ is a flow-dependent constant (i.e. different for pipes and boundary layers since the very large scales behave differently, see, e.g. Monty et al, 2009).

For boundary layers, the preceding analysis is supported by experimental data and $A_{1} \approx 1.25$ appears to fit the data well (Marusic et al, 2013). However, in pipe flows, different spectral scaling has been reported at high Reynolds numbers (Rosenberg et al, 2013) despite logarithmic behaviour of the streamwise turbulence intensity (Marusic et al, 2013; Vallikivi et al, 2015a).

\subsection{A model for the 'crowding' effect of eddies}

Here we propose a simple modification to the upper limit of the constancy in the 2D spectrum for pipe flows to interpret the differences between pipe and boundary 
layer flows. We expect the width of large eddies in pipe flow to be restricted by the geometry of the pipe as illustrated in figure $5(a)$. Therefore let us constrain the upper limit, $\lambda_{U}$, of the constant $k_{x} k_{y} E_{u u}^{2 D}\left(k_{x}, k_{y}\right) / U_{\tau}^{2}$ region such that

$\lambda_{U} / z=C_{2} f_{2}\left(\lambda_{x} / z\right)(1-\beta z / R)$.

This constraint simply reduces $\lambda_{U}$ linearly as the walldistance increases, as illustrated by the arrows in figure $6 ; \beta>0$ is a model constant that determines the strength of the correction. Note that this a low-order correction (i.e. a Taylor series expansion in small $z / R$ for the correction function) designed for conceptual simplicity. More complex constraints could be applied; however, the purpose here is to illustrate that the geometrical constraints imposed by the pipe walls will lead to qualitative differences in a range of statistics that are detailed below.

With this correction, integration of the 2D spectrum to obtain the $1 \mathrm{D}$ spectrum, cf. (6), now gives

$$
\begin{aligned}
\frac{k_{x} E_{u u}\left(k_{x}\right)}{U_{\tau}^{2}} & =\log \left(\frac{C_{2}}{C_{1}}(1-\beta z / R)\right) \\
& =A_{1}+\log (1-\beta z / R) .
\end{aligned}
$$

Since the second term of the right-hand side of (9) is negative (for $\beta>0$ ), the result is a reduction with wall-distance in the level of the $k_{x}^{-1}$ plateau of the $1 \mathrm{D}$ premultiplied spectrum. This means one should not expect a constant plateau that is independent of $z / R$ in the premultiplied spectra of turbulent pipe flows, as has been documented in turbulent boundary layers. Some evidence for this spectral behaviour at high Reynolds numbers is provided in figure 4 of Rosenberg et al (2013) and is further analysed by Vallikivi et al (2015a). It was stated earlier that the conclusions of this paper are not predicated on the existence of the $k_{x}^{-1}$ scaling. Even if $f_{1}\left(\lambda_{x} / z\right)$ and $f_{2}\left(\lambda_{x} / z\right)$ (the upper and lower limits of the constant- $k_{x} k_{y} E_{u u}^{2 D}\left(k_{x}, k_{y}\right) / U_{\tau}^{2}$ region) are different, which will lead to non- $k_{x}^{-1}$ behaviour of the 1D spectrum, the same geometrical correction to the upper limit can still be applied and there will be a consequential difference between turbulent pipe flow and turbulent boundary layer spectra because of this. The aim of this simple model is to explain why there is a difference-the precise nature of the 1D spectrum of the turbulent boundary layer is not critical to this explanation.

Nevertheless, Marusic et al (2013) have shown that the streamwise turbulence intensity in pipe flows and boundary layers follows the same logarithmic behaviour. Making use of (9) and following the same procedure as that used to determine (7) gives the result,

$\frac{\overline{u^{2}}}{U_{\tau}^{2}}=B_{1, \text { Pipe }}-\left[A_{1}+\log (1-\beta z / R)\right] \log \left(\frac{z}{R}\right)$,

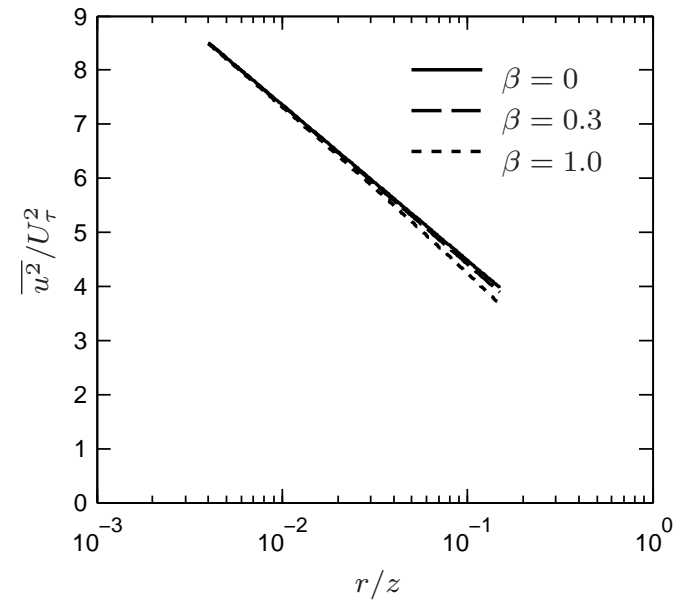

Fig. 7 Streamwise-velocity turbulence variance for selected values of model parameter $\beta$ show that geometrical corrections are negligible in the region $z / R<0.15\left(A_{1} \approx 1.25\right.$, $\left.B_{1, \mathrm{Pipe}} \approx 1.6\right)$.

which means that the slope of the logarithmic region of pipe flows should be different to that of boundary layers. However, the slope is a constant, $A_{1}$, to leading order so the magnitude of the slope change due to the geometrical correction is small. Figure 7 displays the predicted turbulence intensity behaviour for various geometrical-correction strengths, $\beta$. Clearly the change to the slope is not large. In fact, the maximum possible changes are within experimental error through almost the entire log region, so it is unlikely that evidence for the geometrical constraint will be found by solely analysing experimental turbulence intensity data.

\subsection{Second-order structure function}

We now consider modifications to the form of the longitudinal second-order structure function. Using a similar procedure for computing the variance in (7), the dominant contribution to the second-order structure function is partitioned into the three contributions,

$$
\begin{aligned}
\frac{\overline{(\Delta u)^{2}}(r)}{U_{\tau}^{2}}= & 2 \int_{0}^{\infty} \frac{E_{u u}\left(k_{x}\right)}{U_{\tau}^{2}}\left[1-\cos \left(k_{x} r\right)\right] \mathrm{d} k_{x} \\
= & 2 \int_{0}^{1 / \Delta_{E}} \frac{E_{u u}\left(k_{x}\right)}{U_{\tau}^{2}}\left[1-\cos \left(k_{x} r\right)\right] \mathrm{d} k_{x} \\
& +2 \int_{1 / \Delta_{E}}^{1 / z} \frac{E_{u u}\left(k_{x}\right)}{U_{\tau}^{2}}\left[1-\cos \left(k_{x} r\right)\right] \mathrm{d} k_{x} \\
& +2 \int_{1 / z}^{\infty} \frac{E_{u u}\left(k_{x}\right)}{U_{\tau}^{2}}\left[1-\cos \left(k_{x} r\right)\right] \mathrm{d} k_{x},
\end{aligned}
$$

where $r$ is the spatial separation in the streamwise direction. Inserting the models for the $k_{x}^{-1}$ range, $E_{u u}\left(k_{x}\right) / U_{\tau}^{2}=$ $A_{1} / k_{x}$, the large-wavelength range, $E_{u u}\left(k_{x}\right) / U_{\tau}^{2}=\Delta_{E} g_{1}\left(k_{x} \Delta_{E}\right)$ 

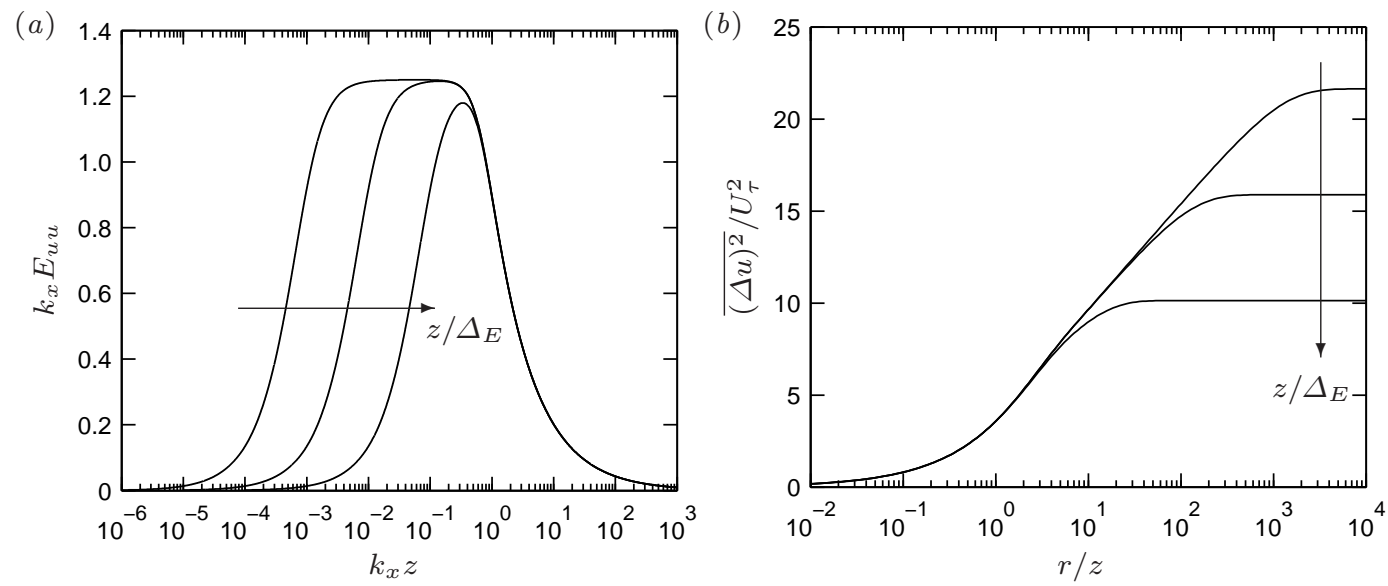

Fig. 8 (a) Model spectra and $(b)$ corresponding second-order structure functions at high Reynolds numbers: $z / \Delta_{E}=$ $0.001,0.01,0.1$. The case when $z / \Delta_{E}=0.001$ is at the bottom of the log region corresponds nominally to $R e_{\tau}=9000000$.

and the small-wavelength range $E_{u u}\left(k_{x}\right) / U_{\tau}^{2}=z g_{2}\left(k_{x} z\right)$, as used in (7), and integrating gives

$$
\begin{array}{rl}
\frac{\overline{(\Delta u)^{2}}(r)}{U_{\tau}^{2}} \sim 2 & 2 \int_{0}^{1} g_{1}(s)\left[1-\cos \left(s \frac{r}{\Delta_{E}}\right)\right] \mathrm{d} s \\
& +2 \int_{1 / \Delta_{E}}^{1 / z} \frac{A_{1}}{k_{x}}\left[1-\cos \left(k_{x} r\right)\right] \mathrm{d} k_{x} \\
& +2 \int_{1}^{\infty} g_{2}(t)\left[1-\cos \left(t \frac{r}{z}\right)\right] \mathrm{d} t \\
\sim & 2 B_{1}-2 \int_{0}^{1} g_{1}(s) \cos \left(s \frac{r}{\Delta_{E}}\right) \mathrm{d} s \\
& -2 \int_{1}^{\infty} g_{2}(t) \cos \left(t \frac{r}{z}\right) \mathrm{d} t \\
& +2 A_{1}\left[\operatorname{Ci}\left(\frac{r}{\Delta_{E}}\right)-\operatorname{Ci}\left(\frac{r}{z}\right)+\log \left(\Delta_{E} / z\right)\right],
\end{array}
$$

where $s=k_{x} \Delta_{E}, t=k_{x} z$ and $\mathrm{Ci}$ is the cosine integral function; recall that $\mathrm{Ci}(\xi) \sim 0$ as $\xi \rightarrow \infty$ and $\mathrm{Ci}(\xi) \sim$ $\gamma+\log (\xi)$ as $\xi \rightarrow 0$, where $\gamma \approx 0.5772$. The $s$ - and $t$-integrals vanish as $r \gg \Delta_{E}$ and $r \gg z$ respectively.

For the turbulent boundary layer, where no geometrical corrections are needed and $\Delta_{E}=\delta$, we have for $z \ll r$ :

$$
\frac{\overline{(\Delta u)^{2}}(r)}{U_{\tau}^{2}} \sim\left\{\begin{array}{cc}
2 C_{1}+2 A_{1}[\gamma+\log (r / z)] & r \ll \delta \\
2 B_{1, \mathrm{BL}}+2 A_{1} \log (\delta / z) & r \gg \delta
\end{array},\right.
$$

cf. Davidson et al (2006b), where $C_{1}=\int_{1}^{\infty} g_{2}(t) \mathrm{d} t$.

For a pipe, we substitute the geometrically corrected $1 \mathrm{D}$ spectrum (9) and the structure function becomes

$$
\begin{aligned}
\frac{\overline{(\Delta u)^{2}}(r)}{U_{\tau}^{2}} \sim & 2 B_{1}-2 \int_{0}^{1} g_{1}(s) \cos \left(s \frac{r}{\Delta_{E}}\right) \mathrm{d} s \\
& -2 \int_{1}^{\infty} g_{2}(t) \cos \left(t \frac{r}{z}\right) \mathrm{d} t \\
& +2\left[A_{1}+\log (1-\beta z / R)\right] \\
& \times\left[\operatorname{Ci}\left(\frac{r}{R}\right)-\operatorname{Ci}\left(\frac{r}{z}\right)+\log (R / z)\right] .
\end{aligned}
$$

The asymptotic behaviour for $z \ll r$ is given by:

$$
\frac{\overline{(\Delta u)^{2}}(r)}{U_{\tau}^{2}} \sim\left\{\begin{array}{ll}
2 C_{1}+2 A_{1}^{\prime}[\gamma+\log (r / z)] & r \ll R \\
2 B_{1, \text { Pipe }}+2 A_{1}^{\prime} \log (R / z) & r \gg R
\end{array},\right.
$$

where $A_{1}^{\prime}=A_{1}+\log (1-\beta z / R)$. Upon comparison with (13), the modification with increasing $z / R$ to the $2 A_{1}$ slope of the $\log r$ behaviour is evident. Note that for both pipe flows and boundary layers, $\overline{(\Delta u)^{2}}(r) \rightarrow 2 \overline{u^{2}}$ as $r \rightarrow \infty$. The $\log r$ behaviour with universal slope $2 A_{1}$ in pipe flow is recovered when $z / R \ll 1$ in (15).

Although these structure-function relations, (12) and (14), qualitatively describe the structure-function behaviour well, plotting the relations reveals large oscillations at $r / z \approx 1$ and $r / z \approx \Delta_{E} / z$ due to the sharp changes at $k_{x} \Delta_{E}=1$ and $k_{x} z=1$ associated with the piecewise-form for $E_{u u}\left(k_{x}\right)$ used in (11) and (14). For this reason, a model spectrum that smoothly blends the different pieces at $k_{x} \Delta_{E}=1$ and $k_{x} z=1$ is used to calculate the model structure function. Specifically, the model spectrum is constructed to behave as $k_{x}^{0}$ (constant) for $k_{x} \Delta_{E}<1 ; k_{x}^{-1}$ for $k_{x} \Delta_{E}>1$ and $k_{x} z<1$; and $k_{x}^{-5 / 3}$ for $k_{x} z>1$. The method for smoothly blending these regions is due to von Kármán (1948) (see also Pope, 2000). In figure 8 , we show these model spectra for $z / \Delta_{E}=0.001,0.01$ and 0.1 without the geometrical correction $(\beta=0)$ along with their corresponding structure functions. Figure 8 demonstrates that extended $\log r$ or $k_{x}^{-1}$ regions require very low values of $z / \Delta_{E}$ and thus extremely high Reynolds numbers.

In figure 9, the model structure function is plotted for turbulent boundary layers and for turbulent pipe flows with a range of geometrical-correction strengths, $\beta$. The notable differences between pipe flows and boundary layers are: 1 ) the structure function peels off from the unperturbed log law at lower $r / z$ for the pipe and 2 ) the apparent slope decreases as $z / R$ increases in the 

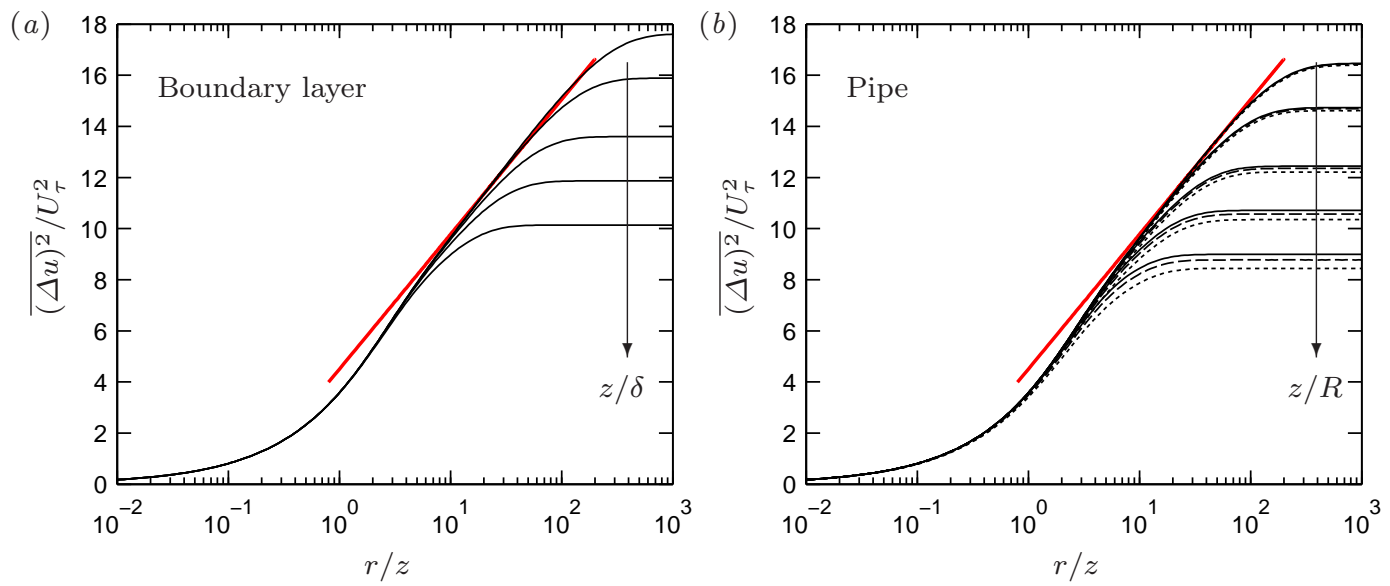

Fig. 9 Second-order structure function modelled at the wall-distances, $z / \Delta_{E}=0.005,0.01,0.025,0.05$ and 0.1, for $(a)$ turbulent boundary layers and $(b)$ turbulent pipe flows with a range of geometrical correction strengths, $\beta=0(-), 0.3(---)$ and $1.0(-\cdots)$. The limiting $r / z \rightarrow \infty$ value of the structure function reduces with $z / \Delta_{E}$.

pipe. In other words, the geometry effect is negligible for small $z / R$. These differences are qualitatively the same as those observed in the experimental data shown in figure 1 and particularly clear for $\beta=1.0$. A consequence of point 2) at lower Reynolds numbers is that the $2 A_{1^{-}}$ slope is never observed, even at the smallest $z / R$ because the $\log$ region is bounded below by $z^{+}>3 R e_{\tau}^{1 / 2}$. For example, if $R e_{\tau} \approx 5000$, the log region is bounded below by $z / R \approx 0.04$, a wall-distance large enough to still be affected by pipe geometry, cf. figures $9(b)$ and $1(b, d)$.

\section{Conclusions}

Second-order structure functions of streamwise velocity are considered in the logarithmic region of wallbounded flows to assess the universality of the scaling behaviours. Measurements in pipes, boundary layers and the atmospheric surface layer indicate that, provided the Reynolds number is sufficiently high and the wall distance is sufficiently small, a $\log r$ behaviour for $\overline{(\Delta u)^{2}}(r) / U_{\tau}^{2}$ is revealed with universal slope $2 A_{1} \approx 2.5$, where $A_{1}$ is the Townsend-Perry constant that is associated with the log law for $\overline{u^{2}} / U_{\tau}^{2}$ and the $k_{x}^{-1}$ spectrum. Deviations from the universal behaviour appear first in pipe flows for nominally $z / R>0.025$, while the boundary layer data show persistent log behaviour for the structure functions further from the wall.

A model for the 'crowding' effect of eddies near the pipe axis is proposed to interpret the observed differences between turbulent pipe flows and turbulent boundary layers. By simply limiting the width of the largest eddies in the 2D spectrum, the model serves to qualitatively demonstrate the link between flow geometry and flow statistics. Consistent with data, the model predicts a shallower slope and an earlier peel-off in the second-order structure function, together with undetectable changes in the streamwise turbulence intensity profile. A more detailed and more accurate model requires extended knowledge of the 2D spectrum at high Reynolds numbers in pipe flows and boundary layers (e.g. Bailey et al, 2008).

The present arguments assume that the scaling of the energy and the bandwidth of the very large scales do not change with Reynolds number, which is consistent with the present data in the range $R e_{\tau} \approx 5000-20000$. However, data at larger Reynolds numbers (Hutchins and Marusic, 2007; Rosenberg et al, 2013) suggest that the influence of the very large scales cannot simply be discounted. Further studies in this regard are ongoing (e.g. Vallikivi et al, 2015a).

Acknowledgements The authors wish to thank the Australian Research Council and ONR under Grant N00014-091-0263 (Program Manager: Ronald Joslin) for the financial support of this research. We also gratefully acknowledge Professor Detlef Lohse for helpful discussions regarding the need for geometrical corrections in pipe flow.

\section{References}

del Álamo JC, Jiménez J, Zandonade P, Moser RD (2004) Scaling of the energy spectra of turbulent channels. J Fluid Mech 500:135-144

Bailey SCC, Hultmark M, Smits AJ, Schultz MP (2008) Azimuthal structure of turbulence in high Reynolds number pipe flow. J Fluid Mech 615:121-138

Davidson PA, Krogstad P (2009) A simple model for the streamwise fluctuations in the log-law region of a boundary layer. Phys Fluids 21:055,105 
Davidson PA, Krogstad P, Nickels TB (2006a) A refined interpretation of the logarithmic structure function law in wall layer turbulence. Phys Fluids 18:065,112

Davidson PA, Nickels TB, Krogstad P (2006b) The logarithmic structure function law in wall-layer turbulence. J Fluid Mech 550:51-60

Högström U, Hunt JCR, Smedman AS (2002) Theory and measurements for turbulence spectra and variances in the atmospheric neutral surface layer. Boundary-Layer Meteorol 103:101-124

Hultmark M, Vallikivi M, Bailey SCC, Smits AJ (2012) Turbulent pipe flow at extreme Reynolds numbers. Phys Rev Lett 108:094,501

Hutchins N, Marusic I (2007) Evidence of very long meandering features in the logarithmic region of turbulent boundary layers. J Fluid Mech 579:1-28

Hutchins N, Nickels TB, Marusic I, Chong MS (2009) Hot-wire spatial resolution issues in wall-bounded turbulence. J Fluid Mech 635:103-136

von Kármán T (1948) Progress in the statistical theory of turbulence. Proc Nat Acad Sci 34:530-539

Klewicki JC, Fife P, Wei T (2009) On the logarithmic mean profile. J Fluid Mech 638:73-93

Kulandaivelu V (2012) Evolution of zero pressure gradient turbulent boundary layers from different initial conditions. PhD thesis, University of Melbourne

Kunkel GJ, Marusic I (2006) Study of the near-wallturbulent region of the high-reynolds-number boundary layer using an atmospheric flow. J Fluid Mech 548:375-402

Marusic I, Monty JP, Hultmark M, Smits AJ (2013) On the logarithmic region in wall turbulence. J Fluid Mech 716:R3

Monty JP (2005) Developments in smooth wall turbulent duct flows. PhD thesis, University of Melbourne

Monty JP, Hutchins N, Ng HCH, Marusic I, Chong MS (2009) A comparison of turbulent pipe, channel and boundary layer flows. J Fluid Mech 632:431-442

Nickels TB, Marusic I, Hafez S, Chong MS (2005) Evidence of the $k_{1}^{-1}$ law in a high-Reynolds-number turbulent boundary layer. Phys Rev Lett 95:074,501

Perry AE, Abell CJ (1975) Scaling laws for pipe-flow turbulence. J Fluid Mech 67:257-271

Perry AE, Chong MS (1982) On the mechanism of wall turbulence. J Fluid Mech 119:173-217

Perry AE, Henbest S, Chong MS (1986) A theoretical and experimental study of wall turbulence. J Fluid Mech 165:163-199

Pope SB (2000) Turbulent Flows. Cambridge University Press

Rosenberg BJ, Hultmark M, Vallikivi M, Bailey SCC, Smits AJ (2013) Turbulence spectra in smooth- and rough-wall pipe flow at extreme Reynolds numbers.
J Fluid Mech 731:46-63

Townsend AA (1976) The Structure of Turbulent Shear Flow. Cambridge University Press

Vallikivi M, Ganapathisubramani B, Smits AJ (2015a) Spectral scaling in boundary layers and pipes at very high Reynolds numbers. J Fluid Mech 771:303-326

Vallikivi M, Hultmark M, Smits AJ (2015b) Turbulent boundary layer statistics at very high Reynolds number. J Fluid Mech In press 


\section{University Library}

\section{- M M N E R VA A gateway to Melbourne's research publications}

Minerva Access is the Institutional Repository of The University of Melbourne

Author/s:

Chung, D;Marusic, I;Monty, JP;Vallikivi, M;Smits, AJ

Title:

On the universality of inertial energy in the log layer of turbulent boundary layer and pipe flows

Date:

2015-07-01

Citation:

Chung, D., Marusic, I., Monty, J. P., Vallikivi, M. \& Smits, A. J. (2015). On the universality of inertial energy in the log layer of turbulent boundary layer and pipe flows. EXPERIMENTS IN FLUIDS, 56 (7), https://doi.org/10.1007/s00348-015-1994-7.

Persistent Link:

http://hdl.handle.net/11343/282843 